\title{
Thromboseprophylaxe in der muskuloskelettalen Chirurgie
}

\author{
Ingrid Pabinger-Fasching - Sabine Eichinger-Hasenauer - Josef Grohs - Josef Hochreiter • \\ Norbert Kastner · Hans Christian Korninger · Sibylle Kozek-Langenecker · Stefan Marlovits · \\ Herwig Niessner · Franz Rachbauer · Peter Ritschl · Christian Wurnig · Reinhard Windhager
}

Eingegangen: 24. April 2013 / Angenommen: 22. Januar 2014 / Online publiziert: 14. Mai 2014

(C) Die Autor(en) 2014. Dieser Artikel ist auf Springerlink.com mit Open Access verfügbar.

Zusammenfassung Muskuloskelettale Eingriffe sind mit einem hohen Risiko für venöse Thrombosen und Pulmonalembolien assoziiert. Die Einführung direkter oraler Antikoagulanzien (DOAK) hat die Möglichkeiten in der Prophylaxe venöser Thromboembolien bei orthopädischen und unfallchirurgischen Eingriffen erweitert. Die Fachgesellschaften für Orthopädie und Orthopädische Chirurgie (ÖGO), Unfallchirurgie (ÖGU), Hämatologie und Onkologie (OeGHO) und für Anaesthesiologie, Reanimation und Intensivmedizin (ÖGARI) tragen dieser Entwicklung Rechnung und haben die Initiative

Sämtliche Abkürzungen stehen, je nach Kontext, entweder für den Einzahl- oder für den Mehrzahlbegriff; Mehrzahl-„,s“ werden für Abkürzungen nicht verwendet.

I. Pabinger-Fasching $(\bowtie) \cdot$ S. Eichinger-Hasenauer, MD Klin. Abt. für Hämatologie und Hämostaseologie,

Univ.-Klinik für Innere Medizin I, MedUni Wien, Wien, Österreich

E-Mail: ingrid.pabinger@meduniwien.ac.at

J. Grohs, MD $\cdot$ R. Windhager, MD

Univ.-Klinik für Orthopädie, MedUni Wien, Wien, Österreich

J. Hochreiter, MD

Orthopädische Abteilung, Krankenhaus der Barmherzigen

Schwestern Linz, Linz, Österreich

N. Kastner, MD

Univ.-Klinik für Orthopädie, MedUni Graz,

Graz, Österreich

H. C. Korninger, MD

Innere Medizin, Unfallkrankenhaus Lorenz Böhler,

Wien, Österreich

S. Kozek-Langenecker, MD

Abt. für Anästhesiologie und Intensivmedizin,

Evangelisches KH Wien,

Wien, Österreich zur Erstellung österreichischer Empfehlungen für die Thromboembolieprophylaxe nach totalem Gelenksersatz von Hüfte und Knie, nach chirurgischer Versorgung von Hüftfrakturen, nach Eingriffen an der Wirbelsäule und nach kleineren orthopädischen und traumatologischen Eingriffen ergriffen. Zudem werden die Pharmakologie der DOAK und die wesentlichen Studiendaten zu jeder einzelnen der derzeit auf dem Markt befindlichen Substanzen - Apixaban, Dabigatran und Rivaroxaban - kurz dargestellt. Den Themen „Antikoagulation und neuroaxiale Blockaden“ und „Bridging“ wurden eigene Abschnitte gewidmet.

\section{S. Marlovits, MD}

Universitätsklinik für Unfallchirurgie Wien,

Wien, Österreich

H. Niessner, MD

Wr. Neustadt., Österreich

F. Rachbauer, MD

Univ.-Klinik für Orthopädie, MedUni Innsbruck, Innsbruck, Österreich

P. Ritschl, MD

1. Orthopädische Abteilung, Orthopädisches Krankenhaus

Gersthof,

Wien, Österreich

C. Wurnig, MD

II. Orthopädische Abteilung, Orthopädisches Spital Speising,

Wien, Österreich 
Schlüsselwörter Venöse Thromboembolie - Thromboembolieprophylaxe $\cdot$ Neue orale Antikoagulanzien · Direkte orale Antikoagulantien - Orthopädie - Bridging . Apixaban · Dabigatran $\cdot$ Rivaroxaban

\section{Prevention of venous thromboembolism in musculoskeletal surgery}

Summary Musculoskeletal surgery is associated with a high risk of venous thrombosis and pulmonary embolism. The introduction of direct oral anticoagulants (DOAK) has broadened the possibilities for prevention of venous thromboembolism in the course of orthopedic and trauma surgery. Addressing this recent development, the Austrian Societies of Orthopedics and Orthopedic Surgery (ÖGO), Trauma Surgery (ÖGU), Hematology and Oncology (OeGHO) and of Anaesthesiology, Reanimation und Intensive Care Medicine (ÖGARI) have taken the initiative to create Austrian guidelines for the prevention of thromboembolism after total hip and knee replacement, hip fracture surgery, interventions at the spine and cases of minor orthopedic and traumatic surgery. Furthermore, the pharmacology of the DOAK and the pivotal trial data for each of the three currently available substances - apixaban, dabigatran, and rivaroxaban - are briefly presented. Separate chapters are dedicated to "anticoagulation and neuroaxial anesthesia" and "bridging".

Keywords Venous thromboembolism - Prevention of thromboembolism - New oral anticoagulants - Orthopedics · Bridging $\cdot$ Apixaban $\cdot$ Dabigatran $\cdot$ Rivaroxaban

$\begin{array}{ll}\text { Abkürzungen } \\ \text { ACCP } & \text { American College of Chest Physicians } \\ \text { AK } & \text { Aortenklappe } \\ \text { aPTT } & \text { Aktivierte partielle Thromboplastinzeit } \\ \text { ASS } & \text { Azetylsalizylsäure } \\ \text { AUC } & \text { Fläche unter der Kurve } \\ \text { AUVA } & \text { Fläche unter der Kurve } \\ \text { Cmax } & \text { Maximale Plasmakonzentration } \\ \text { CT } & \text { Computertomographie } \\ \text { CYP } & \text { Cytochrom-P450-System } \\ \text { DFAK } & \text { Aortenklappe mit Doppelflügel } \\ \text { ESA } & \text { European Society of Anaesthesiology } \\ \text { Hb } & \text { Hämoglobin } \\ \text { HFS } & \text { Chirurgische Versorgung von Hüftfrakturen } \\ \text { HTEP } & \text { Hüft-Totalendoprothese } \\ \text { HWZ } & \text { Halbwertszeit } \\ \text { INR } & \text { International Normalized Ratio } \\ \text { IPCD } & \text { Intermittierendes pneumatisches Kompres- } \\ & \text { sionsgerät } \\ \text { IVC } & \text { Venacava inferior } \\ \text { KI } & \text { Kontraindikation } \\ \text { KrCl } & \text { Kreatininclearance } \\ \text { KTEP } & \text { Knie-Totalendoprothese } \\ \text { LDUH } & \text { Niedrig dosiertes unfraktioniertes Heparin } \\ \text { MK } & \text { Mitralklappe } \\ & \end{array}$

MRT Magnetresonanztomographie

MUW Medizinische Universität Wien

NI Niereninsuffizienz

NMH Niedermolekulare Heparine

DOAK Direkte orale Antikoagulantien

OeGHO Österreichische Gesellschaft für Hämatologie und Onkologie

ÖGARI Österreichische Gesellschaft für Anästhesiologie, Reanimation und Intensivmedizin

ÖGO Österreichische Gesellschaft für Orthopädie und orthopädische Chirurgie

ÖGU Österreichische Gesellschaft für Unfallchirurgie

PCC Prothrombinkomplex-Präparat

PE Pulmonalembolie

P-gp P-Glykoproteintransporter in der Darmwand

PZT Prothrombinzeit

RCT Randomisierte kontrollierte Studie

RF Risikofaktoren

RR Risikoreduktion

RRR Relative Risikoreduktion

$\mathrm{T}_{\max } \quad$ Zeit bis zum Erreichen der maximalen Plasma-

konzentration

TVT Tiefe Venenthrombose

UFH Unfraktioniertes Heparin

VHF Vorhofflimmern

VKA Vitamin-K-Antagonist

VTE Venöse Thromboembolie

\section{Einleitung}

Operationen in der Orthopädie und Traumatologie zählen zu den invasiven Eingriffen mit einem hohen Risiko für venöse Thrombosen und Lungenembolien, und eine Thromboembolieprophylaxe ist seit Jahrzehnten medizinischer Standard bei diesen Eingriffen. Die Verfügbarkeit neuer Antikoagulanzien hat den State of the Art der Thromboseprophylaxe in der orthopädischen Chirurgie und Unfallchirurgie in den letzten Jahren deutlich verändert. Der hier vorliegende Konsensus der österreichischen Fachgesellschaften versucht, dieser Entwicklung Rechnung $\mathrm{zu}$ tragen. Er stellt keine Neubewertung bestehenden Wissens dar, sondern basiert auf internationalen Leitlinien, insbesondere auf den im Februar 2012 in einer neuen Version erschienenen Guidelines des ACCP [1]. Auf dieser Basis wird im vorliegenden Konsensus die Thromboseprophylaxe bei orthopädisch-chirurgischen Eingriffen am Knie, der Hüfte, der Wirbelsäule und bei kleineren Eingriffen sowie Verletzungen der UE unter besonderer Berücksichtigung der neuen Antikoagulanzien diskutiert und es werden an die österreichischen Voraussetzungen und Bedürfnisse adaptierte Empfehlungen gegeben. In weiteren Punkten werden die Themen Thromboseprophylaxe bei rückenmarksnahen Anästhesieverfahren und Bridging dargestellt. 
consensus report

Tab. 1 Pharmakologie der neuen Antikoagulanzien. (Quellen: [2-5])

\begin{tabular}{|c|c|c|c|}
\hline & Apixaban & Dabigatran & Rivaroxaban \\
\hline Wirkmechanismus & Direkte Hemmung von Faktor Xa & Direkte Hemmung von Thrombin & Direkte Hemmung von Faktor Xa \\
\hline Bioverfügbarkeit & $50 \%$ & $6,5 \%$ & $80-100 \%$ \\
\hline Applikationsweg & Oral & Oral & Oral \\
\hline Pro-Drug & Nein & $\mathrm{Ja}$ & Nein \\
\hline Einfluss von Mahlzeiten & Nein & Nein & Nein \\
\hline Renale Ausscheidung & $27 \%$ & $85 \%$ & $33 \%$ \\
\hline $\begin{array}{l}\text { Dosisanpassung bei } \\
\text { eingeschränkter Nieren- } \\
\text { funktion }\end{array}$ & $\begin{array}{l}\text { Bei } \mathrm{KrCl} 30-80 \mathrm{ml} / \mathrm{min} \text { keine Dosis- } \\
\text { anpassung erforderlich; bei } \mathrm{KrCl} \\
15-29 \mathrm{ml} / \mathrm{min} \text { Plasmaspiegel erhöht, } \\
\text { daher mit Vorsicht anzuwenden; bei } \\
\mathrm{KrCl}<15 \mathrm{ml} / \mathrm{min} \text { Anwendung nicht } \\
\text { empfohlen }\end{array}$ & $\begin{array}{l}\text { Erhebliche Verlängerung der HWZ bei Nl; bis zu } \\
\text { einer } \mathrm{KrCl} \text { von } 50 \mathrm{ml} / \mathrm{min} \text { Standarddosis ( } 220 \mathrm{mg} \\
1 \times \text { tgl., in Form von } 2 \mathrm{Kps} \text {. à } 110 \mathrm{mg} \text { ); bei } \mathrm{KrCl} \\
30-50 \mathrm{ml} / \mathrm{min} \text { Dosisreduktion auf } 150 \mathrm{mg} \text { tägl. } \\
\text { (in Form von } 2 \mathrm{Kps} \text { à } 75 \mathrm{mg} \text { ); bei } \mathrm{KrCl}<30 \mathrm{ml} / \\
\text { min Kontraindikation }\end{array}$ & $\begin{array}{l}\text { Bei } \mathrm{KrCl} 30-80 \mathrm{ml} / \mathrm{min} \text { keine } \\
\text { Dosisanpassung erforderlich; bei } \\
\mathrm{KrCl} 15-29 \mathrm{ml} / \mathrm{min} \text { Plasmaspiegel } \\
\text { signifikant erhöht, daher mit Vorsicht } \\
\text { anzuwenden; bei } \mathrm{KrCl}<15 \mathrm{ml} / \mathrm{min} \\
\text { Anwendung nicht empfohlen }\end{array}$ \\
\hline Mediane HWZ & $12 \mathrm{~h}$ & $12-14 \mathrm{~h}$ & $7-11 \mathrm{~h}$ \\
\hline $\mathrm{T}_{\max }$ & $3-4 \mathrm{~h}$ & $0,5-2 \mathrm{~h}$ & $2-4 \mathrm{~h}$ \\
\hline Metabolisierung & $\begin{array}{l}\text { V. a. CYP3A4/5, in geringerem Umfang } \\
\text { CYP1A2, 2C8, 2C9, 2C19 und 2J2; } \\
\text { Substrat von P-gp }\end{array}$ & $\begin{array}{l}\text { Nicht über CYP; Hauptteil unverändert renal aus- } \\
\text { geschieden; Glukuronidierung; Substrat von P-gp }\end{array}$ & $\begin{array}{l}\text { CYP3A4, CYP2J2 sowie CYP-unabhän- } \\
\text { gige Mechanismen; Substrat von P-gp }\end{array}$ \\
\hline Interaktionen ${ }^{\mathrm{a}}$ & $\begin{array}{l}\text { Inhibitoren von CYP3A4 und P-gp } \\
\text { erhöhen AUC und } \mathrm{C}_{\max } \text { v. Apixaban } \\
\text { (z. B. Azol-Antimykotika, HIV-Protease- } \\
\text { inhibitoren); Induktoren von CYP3A4 } \\
\text { und P-gp reduzieren AUC und } \mathrm{C}_{\max } \text { v. } \\
\text { Apixaban (z. B. Rifampicin, Pheny- } \\
\text { toin, Carbamazepin, Phenobarbital, } \\
\text { Johanniskraut) }\end{array}$ & $\begin{array}{l}\text { Bei gleichzeitiger Anwendung von starken P-gp- } \\
\text { Hemmern (z. B. Amiodaron, Verapamil, Chinidin, } \\
\text { Ketoconazol und Clarithromycin) ist eine erhöhte } \\
\text { Dabigatran-Plasmakonzentration zu erwarten. } \\
\text { Bei gleichzeitiger Anwendung von P-gp-Indukto- } \\
\text { ren (wie Rifampicin, Johanniskraut, Carba- } \\
\text { mazepin oder Phenytoin) ist ein verringerter } \\
\text { Dabigatran-Plasmaspiegel zu erwarten }\end{array}$ & $\begin{array}{l}\text { Inhibitoren von CYP3A4 und P-gp } \\
\text { erhöhen AUC und C } \text { max }_{\text {v. Rivaroxaban }} \\
\text { (z. B. Azol-Antimykotika, HIV-Protease- } \\
\text { inhibitoren); Induktoren von CYP3A4 } \\
\text { und P-gp reduzieren AUC und C } \mathrm{C}_{\text {max }} \text { V. } \\
\text { Apixaban (z. B. Rifampicin, Pheny- } \\
\text { toin, Carbamazepin, Phenobarbital, } \\
\text { Johanniskraut) }\end{array}$ \\
\hline
\end{tabular}

\section{Allgemeine Übersicht: neue orale Antikoagulanzien}

Drei verschiedene DOAK - Apixaban, Dabigatran und Rivaroxaban - wurden seit 2008 für die Prophylaxe der VTE bei Hüft- und Kniegelenksersatz zugelassen und werden deshalb im Folgenden eingehender dargestellt.

Der Wirkmechanismus von Apixaban und Rivaroxaban ist eine direkte Hemmung von Faktor Xa, während Dabigatran ein direkter Thrombinhemmer ist.

Tabelle 1 listet wichtige pharmakologische Eckdaten der DOAK auf.

Gemeinsam ist allen DOAK, dass sie - so wie auch die NMH - in einer fixen Dosierung verabreicht werden und kein Gerinnungsmonitoring benötigen. Direkte Vergleichsdaten zwischen den DOAK existieren nicht, da Head-to-Head-Studien fehlen und auch in Zukunft nicht zu erwarten sind.

Für die DOAK gibt es noch kein spezifisches Antidot. Die Wirkung aller drei Substanzen tritt relativ rasch ein. Gewisse Unterschiede bestehen in der Bioverfügbarkeit und dem renal ausgeschiedenen Anteil der Substanz, woraus sich Auswirkungen auf die Notwendigkeit einer Dosisreduktion bei Niereninsuffizienz ergeben (s. Tab. 1).

Den richtigen Zeitpunkt für den Beginn der Prophylaxe mit der jeweiligen Substanz zeigt Tab. 2 .

In diesem Zusammenhang sei darauf hingewiesen, dass es für „Hybridtherapien“, die mancherorts prakti-
Tab. 2 Beginn, Dosierung und Dauer der VTE-Prophylaxe mit DOAK laut Fachinformation. (Quellen: [2-5])

\begin{tabular}{l|l|l|l|l|} 
& $\begin{array}{l}\text { Erste postop. } \\
\text { Dosis nach }(\mathrm{h})\end{array}$ & $\begin{array}{l}\text { Applika- } \\
\text { tionsweg }\end{array}$ & Startdosis & $\begin{array}{l}\text { Erhaltungs- } \\
\text { Tagesdosis }\end{array}$ \\
\hline Dabigatran & $1-4$ & Per os & $\begin{array}{l}110 \mathrm{mg}^{\mathrm{a}} \\
(75 \mathrm{mg})^{\mathrm{a}, \mathrm{c}}\end{array}$ & $\begin{array}{l}220 \mathrm{mg}^{\mathrm{b}} \\
(150 \mathrm{mg})^{\mathrm{c}}\end{array}$ \\
\hline Rivaroxaban & $6-10$ & Per os & $10 \mathrm{mg}$ & $10 \mathrm{mg}$ \\
\hline Apixaban & $12-24$ & Per os & $2,5 \mathrm{mg}$ & $\begin{array}{l}5 \mathrm{mg}(2 \times \text { tägl. } \\
2,5 \mathrm{mg})\end{array}$
\end{tabular}

aWird die Prophylaxe nicht am Tag des Eingriffs begonnen, sollte die Startdosis $220 \mathrm{mg}$ bzw. bei mittelschwerer Niereninsuffizienz $150 \mathrm{mg}$ betragen bIn Form von 2 Kapseln à $110 \mathrm{mg}$

'Bei $\mathrm{KrCl} 30-50 \mathrm{ml} / \mathrm{min}$, Patienten > 75 Jahren sowie Therapie mit Amiodaron, Chinidin oder Verapamil: Startdosis $1 \times 75 \mathrm{mg}$, Erhaltungsdosis $1 \times$ tgl. $150 \mathrm{mg}$

ziert werden - also Beginn präoperativ mit einem NMH und dann 1 bis 2 Tage postoperativ Umstieg auf ein DOAK -, bisher nur sehr wenig Daten gibt [6]. DOAK verändern die Globaltests für die Gerinnung (PTZ, INR, aPTT) in Abhängigkeit von der Plasmakonzentration des Wirkstoffs, ohne damit eine Information über die Intensität der Antikoagulation zu liefern.

Eine Messung der antikoagulatorischen Aktivität mit speziellen Tests (z. B. Hemoclot ${ }^{\mathrm{TM}}$ für Dabigatran sowie Biophen $^{\mathrm{TM}}$ DiXa-I oder Technochrom ${ }^{\mathrm{TM}}$ Anti-Xa für Rivaroxaban; für Apixaban der Rotachrom ${ }^{\circledR}$ Anti-FXa-Test) ist vor einem dringlichen Eingriff oder bei unbeherrschbarer 
Blutung in Erwägung zu ziehen, allerdings können derzeit noch keine Grenzwerte für einen sogenannten „unwirksamen" Spiegel angegeben werden. Jedenfalls kann mit diesen Testen das Vorhandensein der Wirkung, insbesondere von hohen Spiegeln, nachgewiesen werden.

Wenn unter DOAK akute Blutungen auftreten, so ist bei Fehlen spezifischer Antidote nebst dem Standard-Blutungsmanagement die Gabe von Faktorenkonzentraten zu erwägen (z. B. PCC 50 IE/kg Körpergewicht oder rekombinanter Faktor VIIa). Klinische Daten bei Patienten dazu fehlen zwar weitgehend, jedoch gibt es Daten für die Wirkung auf bestimmte Gerinnungstests bei Probanden [7]. In dieser Studie wurde eine Wirkung von PCC im Sinne einer zumindest teilweisen Aufhebung der Wirkung von Rivaroxaban gezeigt. Dabigatran kann dialysiert werden.

$\mathrm{Zu}$ Maßnahmen, mit denen die Aufhebung der Wirkung von DOAK beschleunigt werden kann, stehen bereits internationale und österreichische Leitlinien zur Verfügung $[8,9]$. In diesem Zusammenhang ist zu beachten, dass sich die renale Eliminations-HWZ bei Rivaroxaban und Apixaban bei Patienten mit Niereninsuffizienz nicht wesentlich erhöht - für Apixaban beträgt die $\mathrm{HWZ}$ bei $\mathrm{KrCl}>\mathbf{8 0} \mathrm{ml} / \mathrm{min} \mathrm{15,1} \mathrm{h,} \mathrm{bei} \mathrm{KrCl}<30 \mathrm{ml} /$ min 17,3 h, für Rivaroxaban betragen die analogen HWZWerte 8,3 h und 9,5 h. Für Dabigatran beträgt bei einer $\mathrm{KrCl}>80 \mathrm{ml} / \mathrm{min}$ die $\mathrm{HWZ}$ 13,8 h, während sie sich aufgrund der hohen renalen Ausscheidungsrate von Dabigatran bei einer $\mathrm{KrCl}<30 \mathrm{ml} / \mathrm{min}$ auf $27,5 \mathrm{~h}$ verdoppelt [8]. Zur Dosisreduktion bei eingeschränkter Nierenfunktion siehe Tab. 1 und 2.

Tabelle 3 stellt die österreichischen Zulassungen für $\mathrm{NMH}$, Fondaparinux und DOAK für die VTE-Prophylaxe in der orthopädischen Chirurgie dar.

Tab. 3 Österreichische Zulassungen zur Antikoagulation nach orthopädisch/traumatologischen Eingriffen. (Quellen: [1-5])

\begin{tabular}{|c|c|c|c|c|}
\hline \multirow[b]{2}{*}{ Substanzen } & \multicolumn{4}{|c|}{ Indikationen } \\
\hline & $\begin{array}{l}\text { HTEP/ } \\
\text { KTEP }\end{array}$ & $\begin{array}{l}\text { Hüfttrak- } \\
\text { tur }\end{array}$ & $\begin{array}{l}\text { Eingriffe an der } \\
\text { Wirbelsäule }\end{array}$ & $\begin{array}{l}\text { Kleinere Eingriffe und } \\
\text { Verletzungen der UE }\end{array}$ \\
\hline \multicolumn{5}{|l|}{$\mathrm{NMH}^{\mathrm{a}}$} \\
\hline Bemiparin & $x$ & $x$ & $x$ & $x$ \\
\hline Certoparin & $x$ & $x$ & $x$ & $x$ \\
\hline Dalteparin & $x$ & $x$ & $x$ & $x$ \\
\hline Enoxaparin & $x$ & $x$ & $x$ & $x$ \\
\hline Nadroparin & $x$ & $x$ & $x$ & $x$ \\
\hline \multicolumn{5}{|c|}{ Pentasaccharid } \\
\hline $\begin{array}{l}\text { Fondapa- } \\
\text { rinux }\end{array}$ & $x$ & $x$ & & \\
\hline \multicolumn{5}{|l|}{ DOAK } \\
\hline Apixaban & $x$ & & & \\
\hline Dabigatran & $x$ & & & \\
\hline Rivaroxaban & $x$ & & & \\
\hline \multicolumn{5}{|c|}{$\begin{array}{l}\text { aDie hier genannten NMH sind nicht speziell für die VTE-Prophylaxe einzel- } \\
\text { ner Indikationen wie HTEP oder KTEP, sondern allgemein für die VTE-Pro- } \\
\text { phylaxe zugelassen. Spezielle Dosierungen für Hochrisikopatienten sind der } \\
\text { jeweiligen Fachinformation zu entnehmen }\end{array}$} \\
\hline
\end{tabular}

\section{ACCP-Leitlinien und Zulassungsstatus der Antikoagulanzien in Österreich}

In den aktuellen ACCP-Leitlinien [1] werden für Patienten mit HTEP oder KTEP die DOAK Apixaban, Dabigatran oder Rivaroxaban neben NMH, LDUH, dosisangepassten VKA und ASS mit Grad 1B empfohlen (die Verwendung von IPCD mit Grad 1C), mit einer Minimaldauer von zehn bis 14 Tagen DOAK.

Bei HFS werden NMH, Fondaparinux, LDUH, dosisangepasste VKA oder ASS (alle 1B) sowie IPCD (1C) für mindestens 10-14 Tage empfohlen. NMH sind erste Wahl gegenüber Fondaparinux und LDUH (2B) sowie dosisangepassten VKA und ASS (2C) als Alternativen [1].

Nach anderen großen orthopädischen Eingriffen wird eine Ausdehnung der postoperativen Thromboseprophylaxe bis zu 35 Tage nach dem Eingriff empfohlen, auch wenn der Patient zu diesem Zeitpunkt bereits entlassen ist. Während des stationären Aufenthalts wird laut ACCP die parallele Verwendung einer medikamentösen Thromboseprophylaxe und eines IPCD empfohlen, allerdings mit Empfehlungsgrad 2C [1].

Wenn ein erhöhtes Blutungsrisiko besteht, wird bei großen orthopädischen Eingriffen die Verwendung eines IPCD oder überhaupt keine Thromboseprophylaxe empfohlen (2C). Im vorliegenden Konsensusstatement wird auf einige dieser in den ACCP-Guidelines genannten Therapieoptionen, die in der klinischen Praxis in Österreich, außer in besonderen Fällen, nicht oder fast nicht verwendet werden, nicht weiter eingegangen. Dabei handelt es sich vor allem um UFH, VKA und ASS.

In Österreich etablierte parenterale Therapieoptionen sind einerseits die NMH, andererseits das Pentasaccharid Fondaparinux, die in die folgenden Empfehlungen einbezogen, jedoch nicht im Detail besprochen werden, da die Pharmakologie, der Einsatz und die Datenlage zu diesen Substanzen als weitgehend bekannt vorausgesetzt werden dürfen und bereits in der Vorversion dieses Konsensusstatements ausführlich abgehandelt wurden [10].

Die mediane Zeit bis zum Auftreten einer VTE nach KTEP beträgt 7 Tage, nach HTEP jedoch 17 Tage, bei HTEP wird die präoperative VTE-Inzidenz erst wieder nach drei Monaten erreicht $[11,12]$. Durch eine auf 35 Tage verlängerte Prophylaxedauer nach HTEP lassen sich mit NMH (versus Plazebo) neun symptomatische VTE pro 1.000 Patienten verhindern [13-15]. In den rezenten ACCP-Leitlinien wird hinsichtlich der Dauer nicht mehr zwischen HTEP und KTEP differenziert und es wird bei „Major Orthopedic Surgery“ einer verlängerten Prophylaxe von bis zu 35 Tagen gegenüber einer Prophylaxe von 10-14 Tagen der Vorzug gegeben [1]. Das entspricht auch den österreichischen Empfehlungen von 2007 [10].

Was die Verwendung von Kompressionsstrümpfen (einer definierten Kompressionsklasse) angeht, so gibt es dazu für große orthopädische Eingriffe wenig Daten, da Kompressionsstrümpfe zumeist einen Teil der Standardversorgung darstellten, aber nicht als Intervention untersucht wurden. Eine Studie mit 177 Patienten, die sich einer HTEP oder KTEP unterzogen hatten, zeigte keinen 
eindeutigen Vorteil für Kompressionsstrümpfe als VTEProphylaxe [16]. Studien mit Patienten nach Schlaganfall zeigten einen numerischen, aber nicht statistisch signifikanten Vorteil für Kompressionsstrümpfe in der Verhinderung von VTE, bei gleichzeitigem starken Ansteigen von Hautkomplikationen $[17,18]$.

Die in Österreich zum Zeitpunkt der Konsensuserstellung zugelassenen Antikoagulanzien und deren Indikationen sind in Tab. 3 zusammengefasst.

\section{Studienübersicht: DOAK und KTEP}

Die publizierten Studien mit DOAKS, bei denen diese hinsichtlich Effektivität und Sicherheit mit niedermolekularem Heparin verglichen werden, sind ausführlich und zusammenfassend in einer rezenten Publikation dargestellt [19]. In der Folge wird hier nur kurz auf die wichtigsten Aspekte hinsichtlich Design und Studienergebnisse eingegangen.

Apixaban wurde in der ADVANCE-1-Studie bei 3.195 Patienten mit KTEP mit Enoxaparin verglichen [20]. Apixaban wurde in einer Dosis von $2 \times 2,5 \mathrm{mg}$ täglich, Enoxaparin in der nordamerikanischen Dosierung von $2 \times 30 \mathrm{mg}$ täglich verabreicht, beide Regime begannen 12-24 h postoperativ. Der primäre Endpunkt war ein Compositum aus asymptomatischer und symptomatischer TVT, PE und Tod jeglicher Ursache. 908 Patienten standen nicht für die Wirksamkeitsanalyse zur Verfügung, und die Ereignisrate war insgesamt viel niedriger als angenommen. Mit 9,0\%, die unter Apixaban den primären Endpunkt erreichten, vs. 8,8\% unter Enoxaparin erreichte Apixaban die Nichtunterlegenheitskriterien nicht, zeigte jedoch eine signifikant niedrigere Blutungsrate als Enoxaparin.

Im Gegensatz dazu stehen die Ergebnisse der ADVANCE-2-Studie bei 3.057 KTEP-Patienten [21]. Hier erfolgte der Vergleich von $2 \times 2,5 \mathrm{mg}$ Apixaban mit $1 \times 40 \mathrm{mg}$ Enoxaparin täglich, wobei hier Enoxaparin $12 \mathrm{~h}$ präoperativ begonnen wurde, Apixaban 12-24 h postoperativ. In dieser Studie zeigte sich unter Apixaban ein signifikant niedrigeres Risiko für den primären Endpunkt, der identisch war mit jenem aus ADVANCE-1, und die Blutungsraten waren nicht signifikant unterschiedlich.

Dabigatran wurde in der RE-MODEL-Studie bei 2.076 Patienten, die sich einer KTEP unterziehen mussten, mit dem NMH Enoxaparin verglichen [22]. Dabigatran wurde in einer Dosierung von 150 mg oder 220 mg einmal täglich verabreicht, beginnend mit einer halben Tagesdosis ein bis $4 \mathrm{~h}$ postoperativ. Enoxaparin wurde in einer Tagesdosis von $40 \mathrm{mg}$ gegeben, beginnend am Abend vor dem Eingriff. Sowohl hinsichtlich des primären Wirksamkeitsendpunkts (VTE oder Tod) als auch hinsichtlich der Blutungsraten erwiesen sich beide Dabigatran-Dosierungen als nicht unterlegen gegenüber Enoxaparin.

In der RE-MOBILIZE-Studie wurde bei 1.896 KTEP-Patienten Dabigatran (150 oder 220 mg) ebenfalls mit Enoxaparin verglichen, letzteres allerdings in einer Dosierung von $2 \times 30 \mathrm{mg}$ täglich (nordamerikanisches Dosierungs- schema) [23]. In dieser Studie zeigten sich beide Dabigatran-Dosierungen hinsichtlich der VTE-Raten dem Enoxaparin-Regime signifikant unterlegen; hingegen waren die Blutungsraten vergleichbar. In einer Metaanalyse, in die neben den beiden genannten Arbeiten auch eine Studie zu Dabigatran vs. Enoxaparin bei HTEP einfloss, zeigte sich kein signifikanter Unterschied zwischen Dabigatran und Enoxaparin, weder bei den Wirksamkeitsendpunkten noch bei den Blutungsraten [24].

Rivaroxaban wurde in der RECORD-3-Studie bei 2.531 Patienten mit KTEP mit Enoxaparin verglichen [25]. Die Rivaroxaban-Dosis betrug $10 \mathrm{mg}$ täglich, beginnend 6 bis $8 \mathrm{~h}$ postoperativ; Enoxaparin wurde in der Standarddosis von einmal täglich $40 \mathrm{mg}$ gegeben, beginnend $12 \mathrm{~h}$ vor dem Eingriff. Der primäre Endpunkt war VTE (TVT oder PE) oder Tod 13 bis 17 Tage postoperativ. Hier zeigte sich eine signifikante Überlegenheit von Rivaroxaban hinsichtlich des primären Endpunkts sowie sekundärer Endpunkte (große VTE, symptomatische VTE) bei gleichen Blutungsraten.

Ein ähnliches Resultat zeigte die RECORD-4-Studie, in der bei 3.148 KTEP-Patienten $10 \mathrm{mg}$ Rivaroxaban mit $2 \times 30 \mathrm{mg}$ Enoxaparin verglichen wurden [26]. Auch hier erwies sich Rivaroxaban in der Wirkung signifikant überlegen, während sich die Blutungsraten nicht signifikant unterschieden.

Die bereits erwähnte Metaanalyse verglich auch gepoolte Daten zu Rivaroxaban versus Enoxaparin, wobei auch hier neben den beiden genannten Arbeiten eine dritte Studie (RECORD-1) mit HTEP-Patienten einfloss [24]. Hier zeigte sich, dass unter Enoxaparin im Vergleich mit Rivaroxaban zwar ein doppelt so hohes Risiko für symptomatische VTE oder Tod bestand, dafür aber ein signifikant niedrigeres Blutungsrisiko.

\section{Studienübersicht: DOAK und HTEP}

Auch hier soll auf eine rezente Übersichtsarbeit, die ausführlich die Effektivität und Sicherheit der DOAKS im Vergleich zu Enoxaparin zusammenfassend darstellt, hingewiesen werden [19].

Apixaban wurde in der ADVANCE-3-Studie in einer Dosis von $2 \times 2,5 \mathrm{mg}$ täglich mit Enoxaparin $40 \mathrm{mg}$ über 5 Wochen bei 5.407 HTEP-Patienten verglichen [27]. Auch hier war der primäre Endpunkt definiert als Gesamt-VTE plus Tod. Er trat bei 1,4\% unter Apixaban und bei 3,9\% unter Enoxaparin auf, ein Ergebnis, das nicht nur hinsichtlich Nichtunterlegenheit, sondern auch hinsichtlich Überlegenheit von Apixaban statistisch signifikant war. Der kombinierte Sicherheitsendpunkt von schweren und klinisch signifikanten Blutungen zeigte keinen signifikanten Unterschied zwischen den Gruppen. Allerdings erfolgte auch hier (wie bei Rivaroxaban, s. weiter unten) der Hb-Vergleich mit dem postoperativen, nicht mit dem präoperativen Wert.

Dabigatran wurde in der RENOVATE-Studie bei 3.494 HTEP-Patienten mit Enoxaparin verglichen [28]. Die Studie hatte drei Arme, die einmal täglich entweder $220 \mathrm{mg}$ 
Dabigatran, 150 mg Dabigatran oder 40 mg Enoxaparin erhielten. Der primäre Endpunkt war ein Compositum aus asymptomatischen und symptomatischen VTE sowie Tod. Dieser Endpunkt trat unter $220 \mathrm{mg}$ Dabigatran bei 6,0\%, unter Dabigatran $150 \mathrm{mg}$ bei $8,6 \%$ und unter Enoxaparin bei $6,7 \%$ auf. Dies bedeutet für beide Dabigatran-Dosierungen den Beweis der Nichtunterlegenheit gegenüber Enoxaparin nach den definierten Kriterien. In den Raten großer Blutungen fand sich jeweils kein signifikanter Unterschied zwischen den Dabigatran-Gruppen und der Enoxaparin-Gruppe.

Aufgrund dieser Ergebnisse wurde in der RENOVATEII-Studie nur noch die Dosis von $220 \mathrm{mg}$ Dabigatran bei 2.055 HTEP-Patienten mit $40 \mathrm{mg}$ Enoxaparin verglichen [29]. Der primäre Endpunkt war gleich definiert wie in RENOVATE und trat unter Dabigatran bei 7,7\%, unter Enoxaparin bei 8,8\% auf, was auch in diesem Fall Nichtunterlegenheit $(p<0,0001)$ von Dabigatran bedeutete. Die Rate schwerer Blutungen betrug unter Dabigatran 1,4\%, unter Enoxaparin 0,9\%, was nicht statistisch signifikant war.

Neben der bereits im Kapitel „Studienübersicht: DOAK und KTEP“ erwähnten Metaanalyse [24], in der sich weder bei den Wirksamkeitsendpunkten noch bei den Blutungsraten ein signifikanter Unterschied zwischen Dabigatran und Enoxaparin zeigte, gab es noch eine weitere Metaanalyse, in welche die beiden RENOVATE-Studien sowie zwei Studien zu Dabigatran vs. Enoxaparin bei KTEP einflossen [30]. Darin wurde für die 220 mg-Dosis von Dabigatran gleiche Wirksamkeit bei gleichem Blutungsrisiko gegenüber $40 \mathrm{mg}$ Enoxaparin gezeigt. $150 \mathrm{mg}$ Dabigatran reduzierten im Vergleich zu $2 \times 30 \mathrm{mg}$ Enoxaparin asymptomatische TVT weniger stark, verursachten aber auch weniger Blutungen.

Rivaroxaban wurde in der RECORD-1-Studie bei 4.541 HTEP-Patienten in einer Dosis von $10 \mathrm{mg}$ täglich mit 40 mg Enoxaparin verglichen [31]. Der primäre Endpunkt war auch hier ein Compositum aus Gesamt-VTE und Tod. Die Behandlungsdauer betrug 5 Wochen. Der primäre Endpunkt trat unter Rivaroxaban signifikant seltener auf als unter Enoxaparin (1,1 vs. 3,7\%, ARR $2,6 \%, p<0,001)$ [31]. Schwere VTE waren unter Rivaroxaban signifikant seltener als unter Enoxaparin (0,2 vs. $2,0 \%$, ARR $1,7 \%, p<0,001)$. Die Rate schwerer Blutungen zeigte keinen signifikanten Unterschied zwischen beiden Gruppen ( 0,3 vs. $0,1 \%, p=0,18)$.

Die RECORD-2-Studie verglich bei 2.509 HTEP-Patienten eine 5 Wochen dauernde VTE-Prophylaxe mit Rivaroxaban mit einer nur knapp 2 Wochen dauernden VTE-Prophylaxe mit $40 \mathrm{mg}$ Enoxaparin [32]. Es zeigte sich demgemäß ein deutlicherer Unterschied beim primären Endpunkt zugunsten von Rivaroxaban (2,0 vs. 9,3\%, ARR $7,3 \%, p<0,0001)$. Die Blutungsraten der beiden Gruppen unterschieden sich nicht signifikant.

Allerdings wurden die beiden RECORD-Studien bezüglich der Definition von Blutungskomplikationen kritisiert, einerseits weil Blutungen am Operationsort nicht als Komplikation gewertet wurden, andererseits weil der $\mathrm{Hb}$-Abfall durch Blutungen aufgrund des post- operativen Werts und nicht des präoperativen Ausgangswerts bestimmt wurde. Zudem fand sich auch in der bereits im Punkt 3 erwähnten Metaanalyse unter Rivaroxaban ein signifikant erhöhtes Blutungsrisiko gegenüber Enoxaparin [24].

Eine rezente gepoolte Analyse von zehn randomisierten, kontrollierten Studien (RCT) der Phase III, in denen bei insgesamt 32.144 Patienten mit HTEP oder KTEP ein DOAK mit Enoxaparin $1 \times 40 \mathrm{mg}$ verglichen wurde, fanden sich folgende Ergebnisse [19]. Als Wirksamkeitsendpunkte wurde VTE insgesamt plus Gesamtmortalität, schwere VTE und proximale TVT gewählt. Sicherheitsendpunkte waren schwere und klinisch relevante Blutungen. Insgesamt fand sich für DOAK im Vergleich zu Enoxaparin für VTE plus Tod eine RRR von $29 \%$, für schwere VTE eine RRR von $41 \%$ und für proximale TVT eine RRR von $49 \%$. Die stärkste RRR zeigte Rivaroxaban $(0,50)$, gefolgt von Apixaban $(0,63)$ und Dabigatran $(1,02)$, allerdings bei beträchtlicher Heterogenität der inkludierten Studien. Für schwere Blutungen $(1,04)$ und klinisch relevante Blutungen $(1,02)$ fand sich insgesamt kein Unterschied zu Enoxaparin. Rivaroxaban zeigte einen Trend zu mehr schweren Blutungsepisoden als Enoxaparin $(1,88)$, während Apixaban $(0,81)$ das niedrigste Risiko für schwere Blutungen aufwies.

\section{Chirurgische Versorgung von Hüftfrakturen}

Zum Vergleich der DOAK mit Enoxaparin oder einem anderen NMH in der Indikation HFS liegen derzeit keine Daten vor. Die größten Studien wurden mit Fondaparinux im Vergleich zu Enoxaparin durchgeführt [33, 34], wobei eine überlegene Wirkung von Fondaparinux gegenüber Enoxaparin bei nicht signifikant erhöhtem Blutungsrisiko gezeigt wurde.

Empfehlungen zu totalem Hüft- und Kniegelenksersatz, Hüftfraktur und anderen großen orthopädisch-chirurgischen Eingriffen

Bei elektivem totalem Hüft- und Kniegelenksersatz (HTEP und KTEP) sind NMH, Fondaparinux und die DOAK Dabigatran, Rivaroxaban und Apixaban als Medikamente erster Wahl zu betrachten.

Bei chirurgischer Versorgung von Hüftfrakturen (HFS) werden NMH und Fondaparinux als erste Wahl empfohlen, wobei die Datenlage für Fondaparinux eine gewisse Überlegenheit zeigt. Zu den DOAK Apixaban, Dabigatran und Rivaroxaban liegen in dieser Indikation keine Daten vor.

$\mathrm{Da}$ es für eine Reihe anderer großer orthopädisch-chirurgischer Eingriffe (z. B. beidseitige HTEP oder KTEP, Revisionsoperationen etc.) keine Daten zur VTE-Prophylaxe mit neueren Substanzen gibt, müssen hinsichtlich des prophylaktischen Vorgehens Analogieschlüsse gezogen werden. NMH sind dabei als Medikamente erster Wahl zu betrachten. Dabei ist allerdings je nach Grundkrankheit und Art 
des Eingriffs die Abschätzung des Blutungsrisikos in die Überlegungen einzubeziehen.

Dauer der Prophylaxe Einer verlängerten Prophylaxe von bis zu 35 Tagen wird gegenüber einer Prophylaxe von nur 10-14 Tagen der Vorzug gegeben.

Der zusätzliche Einsatz von Kompressionsstrümpfen kann erwogen werden, es gibt aber dazu keine gesicherten, positiven Daten.

Bei Patienten mit hohem Blutungsrisiko soll die Verwendung eines IPCD bzw. keiner Prophylaxe gegenüber einer medikamentösen Prophylaxe in Erwägung gezogen werden.

\section{Eingriffe an der Wirbelsäule}

Zur VTE-Prophylaxe bei chirurgischen Eingriffen an der Wirbelsäule gibt es sehr wenig wissenschaftliche Daten. Bei elektiven Wirbelsäuleneingriffen wird die Rate an symptomatischen TVT mit 3,7\%, die Rate an symptomatischen PE mit 2,2\% angegeben [35]. Das bedeutet, dass Wirbelsäuleneingriffe ein hohes Risiko für symptomatische VTE mit sich bringen, annähernd jenem nach HTEP oder KTEP vergleichbar [36, 37]. Im Gegensatz dazu besteht für asymptomatische, phlebographisch diagnostizierte TVT in diesem Patientengut einer Untersuchung zufolge ein Risiko von $18 \%$, was deutlich niedriger ist als bei Patienten mit HTEP oder KTEP [36-38]. Tabelle 4 zeigt Risikofaktoren für VTE bei elektiven Wirbelsäuleneingriffen.

Sechs RCT untersuchten verschiedene Methoden der VTE-Prophylaxe bei Wirbelsäulenoperationen. Diese Studien weisen eine Reihe methodischer Probleme hinsichtlich der Fallzahlen, der Verblindung und der diagnostischen Methodik bezüglich asymptomatischer TVT auf. Eine Metaanalyse fasste diese und einige andere Studien (18 RCT und 12 Kohortenstudien mit insgesamt 7.779 Patienten) zur VTE-Prophylaxe bei neurochirurgischen Eingriffen zusammen [39]. Dabei zeigte sich durch NMH ein relatives Risiko von 0,60 gegenüber Kompressionsstrümpfen, durch IPCD eine RR von 0,41 gegenüber Plazebo. In Head-to-Head-Studien fand sich bezüglich intrakranieller Blutungen kein statistisch signifikanter Unterschied zwischen $\mathrm{NMH}$ und nicht medikamentösen Methoden. Die gepoolten

Tab. 4 VTE-Risikofaktoren bei elektiven Wirbelsäulenoperationen. (Quellen: [36, 37])

Fortgeschrittenes Alter ( $\geq 60 \mathrm{a}$ )
Eingriffe an der Lendenwirbelsäule
Vorderer Zugang (Kompression der V. cava?)
Malignomoperation
Lange Operationsdauer ( $\geq 4 \mathrm{~h}$ )
Reduzierte prä- und postoperative Mobilität
VTE-Anamnese

Fortgeschrittenes Alter ( $\geq 60 \mathrm{a})$

Eingriffe an der Lendenwirbelsäule

Vorderer Zugang (Kompression der V. cava?)

Malignomoperation

Reduzierte prä- und postoperative Mobilität

VTE-Anamnese
Raten intrakranieller sowie kleiner Blutungen waren jedoch unter Heparin-basierter Prophylaxe höher als unter nicht-Heparin-basierten Methoden.

Die ACCP-Guidelines empfehlen bei elektiven chirurgischen Eingriffen an der Wirbelsäule, sofern keine zusätzlichen Risikofaktoren (Tab. 4) bestehen, die Anwendung einer mechanischen Prophylaxe (vorzugsweise IPCD) anstelle einer medikamentösen VTE-Prophylaxe (2C) [40].

Bei Patienten mit hohem Risiko (einschließlich Malignompatienten und solchen, bei denen ein anteroposteriorer Zugang gewählt wird) wird die Zugabe einer medikamentösen Prophylaxe (NMH oder UFH) zur mechanischen Prophylaxe empfohlen, sobald eine adäquate Hämostase gesichert ist und das Blutungsrisiko sinkt (2C) [40].

Auch für VTE-Prophylaxe bei Operationen nach Traumata der Wirbelsäule gibt es zwar eine Reihe von Studien, die jedoch ähnliche methodische Probleme aufweisen - qualitativ hochwertige Daten fehlen auch hier.

Dass das VTE-Risiko bei Patienten mit Wirbelsäulentrauma auch innerhalb der Gruppe der Trauma-Patienten besonders hoch ist, zeigte eine Studie mit 395 Trauma-Patienten [41]. 71\% der Patienten erhielten eine VTE-Prophylaxe (LDUH oder mechanisch), $29 \%$ nicht. Insgesamt lag die sonographisch diagnostizierte VTE-Rate bei Patienten ohne Prophylaxe bei 8,8\%, mit Prophylaxe bei $2,9 \%$. Patienten mit neurologischen Verletzungen hatten mit $14 \%$ insgesamt eine signifikant höhere VTE-Rate als Patienten ohne neurologisches Defizit mit 2,7\% $(p<0,001)$. Patienten mit spinalen Traumata ohne Prophylaxe wiesen eine VTE-Rate von $27,3 \%$, mit Prophylaxe waren es $10,3 \%$.

Die ACCP definiert daher Patienten mit schweren Wirbelsäulentraumen und/oder akuten Rückenmarksverletzungen als VTE-Hochrisikopatienten und empfiehlt bei dieser Gruppe die Kombination von pharmakologischer (LDUH, NMH) und mechanischer Prophylaxe (IPCD), sofern letztere nicht durch Verletzungen der unteren Extremitäten verhindert wird (2C) [40].

Zur Wirksamkeit von Fondaparinux und DOAK gibt es in diesen Indikationen noch keine Untersuchungen und keine Zulassung. Ihre Anwendung würde einen Off-Label-Gebrauch darstellen.

\section{Empfehlungen zu Eingriffen an der Wirbelsäule} Patienten, bei denen Eingriffe an der Wirbelsäule durchgeführt werden, sollten bei erhöhtem VTERisiko (elektive Eingriffe mit Risikofaktoren, Wirbelsäulen- und Rückenmarksverletzungen) eine VTE-Prophylaxe mit NMH und - nach Möglichkeit - mechanischen Kompressionsmethoden erhalten. Die Dauer der Prophylaxe hängt von der Art des Eingriffs ab (z. B. Dekompressionen: 2 Wochen, Sta- 
bilisierungen: 5 Wochen, bei Bettlägerigkeit oder Paraparesen: mehrere Monate).

Als zweite Wahl kommen - vor allem für längerfristige Antikoagulationen in besonderen klinischen Situationen - VKA in Frage.

Der zusätzliche Einsatz von Kompressionsstrümpfen sollte erwogen werden.

\section{Kleinere Eingriffe im Bereich des Bewegungsapparates an der UE}

Für die VTE-Prophylaxe bei kleineren Eingriffen im Bereich des Bewegungsapparates an der unteren Extremität, wie z. B. Kniearthroskopien, wurden neben den aktuellen ACCP-Guidelines [1] auch ein österreichischer Konsensus aus 2007 [10] sowie Leitlinien, die im Bereich der AUVA erarbeitet wurden [42], herangezogen. Diese werden im Folgenden gegenübergestellt.

Arthroskopische Operationen am Knie können sehr unterschiedliche Eingriffe wie z. B. Meniskus(teil) resektionen, Plicaresektionen, Meniskusrefixationen, Knorpeloperationen oder Kreuzbandersatzoperationen sein. Ein Cochrane-Review von vier Studien mit 527 Patienten fand, dass NMH im Vergleich zu Plazebo plus Kompressionsstrümpfen bei Arthroskopien sechs VTE pro 1.000 Patienten verhindern [43]. Es handelte sich allerdings ausschließlich um UnterschenkelTVT. In einer rezenteren Studie mit 1.761 Patienten wurden ebenfalls NMH mit Kompressionsstrümpfen verglichen [44]. In dieser Studie wurde die Zahl von Ereignissen im kombinierten Endpunkt (asymptomatische und symptomatische TVT sowie Tod) durch NMH gegenüber Kompressionsstrümpfen signifikant gesenkt. In der NMH-Gruppe traten 15 symptomatische TVT weniger auf als in der Kompressionsgruppe ( $n=6$ vs. 21 ), jedoch auch drei schwere Blutungen und eine PE mehr.

Die ACCP-Guidelines empfehlen aufgrund dieser Datenlage bei Patienten ohne VTE-Anamnese keine VTE-Prophylaxe bei Arthroskopien. Allerdings wird darauf hingewiesen, dass es vernünftig erscheinen mag, die Daten für die VTE-Prophylaxe aus anderen Hochrisikogruppen auf Arthroskopiepatienten mit Risikofaktoren (insbesondere VTE-Anamnese) zu extrapolieren [1].

Auch die österreichischen Leitlinien empfehlen in diesem Fall eine Prophylaxe mit NMH nur bei Risikofaktoren oder größerem Eingriff [10]. Die AUVA empfiehlt eine VTE-Prophylaxe ebenfalls bei RF sowie bei bestimmten Eingriffen (Kreuzbandersatzoperation, etc.) oder bei Teilbelastung [42].

Zur Dauer der Prophylaxe ist zu sagen, dass nur wenige vergleichenden Studien mit unterschiedlicher Dauer durchgeführt wurden [45]. Die deutsche S 3-Leitlinie gibt eine konkrete Empfehlung für die Dauer mit einem Empfehlungsgrad mittlerer Stärke („sollte“), die unten aufgeführt ist. Eine Prophylaxe mit NMH sollte zumindest bis zum Erreichen einer normalen freien
Gelenksbeweglichkeit und einer Belastung der unteren Extremität von mindestens $20 \mathrm{~kg}$ durgeführt werden und dies mindestens für die Dauer von 7 Tagen.

\section{Empfehlungen zu Arthroskopien}

Bei Patienten mit Arthroskopie, bei denen keine Risikofaktoren, insbesondere keine VTE-Anamnese besteht, wird eine medikamentöse VTE-Prophylaxe nicht empfohlen.

Wenn Risikofaktoren von Seiten des Patienten (z. B. VTE-Anamnese) oder von Seiten des Eingriffs (z. B. Kreuzbandoperationen) bestehen, sollte jedenfalls eine medikamentöse VTE-Prophylaxe mit NMH durchgeführt werden.

Dauer der Prophylaxe Die Prophylaxe sollte bis zum Erreichen einer normalen freien Gelenksbeweglichkeit und einer Belastung der unteren Extremitätvon mindestens $20 \mathrm{~kg}$, für die Dauer von mindestens 7 Tagen durchgeführt werden.

\section{Verletzungen der UE}

Bei Verletzungen der unteren Extremitäten ist die Datenlage noch schlechter, vor allem deshalb, weil die Heterogenität der Verletzungen in den Studien sehr groß ist. Ein Cochrane-Review, das sechs Studien mit 1.490 Patienten umfasste, fand zwar eine signifikante Abnahme von VTE unter NMH im Vergleich zu Plazebo/Kompressionsstrümpfen (1 PE und 16 symptomatische TVT pro 1.000 Patienten weniger), dem stand aber eine Zunahme von vier schweren Blutungen pro 1.000 Patienten gegenüber [46].

Auch hier empfehlen die ACCP-Guidelines bei Patienten ohne Risikofaktoren mit Unterschenkelverletzungen, die eine Immobilisation erfordern, keine VTE-Prophylaxe [1]. Die österreichischen Leitlinien empfehlen im Gegensatz dazu jedenfalls bei Patienten mit Frakturen bis zur Gipsabnahme sehr wohl eine VTE-Prophylaxe [10]. Die AUVA-Leitlinien fordern ebenfalls eine VTEProphylaxe bis zur Gipsabnahme und bei Patienten ohne Gips dann, wenn nicht zumindest eine Teilbelastung von $20 \mathrm{~kg}$ und eine Beweglichkeit des oberen Sprunggelenks von $20^{\circ}$ möglich ist. Bei stumpfen Weichteiltraumen der UE (Muskeltrauma) wird eine Prophylaxe mit NMH bis zum Erreichen der Vollbelastung empfohlen.

Zur Wirksamkeit von Fondaparinux und DOAK gibt es in diesen Indikationen noch keine Untersuchungen und keine Zulassung. In Analogie zu den Daten für die zugelassenen Indikationen lässt sich jedoch schließen, dass diese Substanzen auch in den hier genannten Indikationen wirksam sind. Ihre Anwendung würde jedoch einen Off-Label-Gebrauch darstellen. 


\begin{abstract}
Empfehlungen zu Ruhigstellung der unteren Extremität nach Verletzung

Es ist festzuhalten, dass bei erwachsenen Patienten mit Ruhigstellung der unteren Extremität nach Verletzung die VTE-Prophylaxe in europäischen Ländern zur Routineversorgung zählt. Patienten mit Gipsverbänden oder ähnlichen Immobilisationen der unteren Extremität nach Verletzungen sollten zumindest bis Gipsabnahme eine VTE-Prophylaxe erhalten, insbesondere dann, wenn zusätzliche Risikofaktoren bestehen.

$\mathrm{NMH}$ stellen in all diesen Indikationen die erste Wahl dar.

Der zusätzliche Einsatz von Kompressionsstrümpfen kann erwogen werden.
\end{abstract}

\section{Antikoagulation und neuroaxiale Blockaden}

Spinale Hämatome sind bei nicht antikoagulierten Patienten selten - wenn sie jedoch auftreten, können sie dramatische neurologische Konsequenzen nach sich ziehen. Bei Patienten ohne Antikoagulation wird die Inzidenz von spinalen Hämatomen niedrig angegeben: 1:150.000 bei Epiduralanästhesie und 1:220.000 bei Spinalanästhesie [47]. Auffallend gehäuft wurden spinale Hämatome mit 1:3.100 nach Epiduralanästhesie während der U.S.-Markteinführung von niedermolekularen Heparinen zur VTE-Prophylaxe in der erhöhten Dosierung beobachtet [48]. Unter Gerinnungshemmung wurden auch in anderen Publikationen wesentlich höhere Inzidenzen angegeben - bis zu 1:18.000 bei Epiduralanästhesie und 1:156.000 bei Spinalanästhesien [49]. Inzidenzzahlen aus aktuellen Registerdaten bestätigen die Größenordnung des Problems [48, 50-55].

Das Risiko eines spinalen Hämatoms war bei Spinalanästhesien bei Schwangeren mit 1:200.000 wesentlich geringer, als bei nicht-schwangeren Frauen nach Kniegelenksersatz mit 1:3.600 [49]. Daten aus dem deutschen Register für Regionalanästhesie bestätigen die unterschiedlichen Risiken mit 1:562.600 bei geburtshilflichen Epiduralanästhesien und 1:11.250 bei nicht-geburtshilflichen Epiduralanästhesien [56].

Es gibt eine Reihe von Risikofaktoren für spinale Hämatome, die in Tab. 5 aufgezählt sind.

Tab. 5 Risikofaktoren für spinale Hämatome. (Quelle: [57])

Fehlen von Leitlinien
Weibliches Geschlecht
Technik
spinal < epidural
Single Shot < Katheter
Blutige Punktion
Anatomische Veränderungen (z. B. Spina bifida)
Antikoagulation
Eingeschränkte Nierenfunktion

Tab. 6 Antikoagulanzien und neuroaxiale Anästhesie ${ }^{a}$. (Quellen: [58, 59])

\begin{tabular}{|c|c|c|c|c|}
\hline \multirow[t]{2}{*}{ Substanz } & \multicolumn{2}{|c|}{ Karenz vor Intervention (h) } & \multicolumn{2}{|c|}{$\begin{array}{l}\text { Folgedosis nach Punktion } \\
\text { bzw. Katheterentfernung (h) }\end{array}$} \\
\hline & ESA & ÖGARI & ESA & ÖGARI \\
\hline $\mathrm{NMH}^{\mathrm{b}}$ & 12 & 11 & 4 & 2 \\
\hline Fondaparinux $x^{b}$ & $36-42$ & 36 & $6-12$ & 6 \\
\hline Rivaroxaban ${ }^{b}$ & $22-26$ & 16 & $4-6$ & 3 \\
\hline Apixaban & $20-30$ & 23 & $4-6$ & 3 \\
\hline Dabigatran & $\mathrm{Kl}^{\mathrm{c}}$ & $(26)^{d}$ & I & 4 \\
\hline \multicolumn{5}{|c|}{$\begin{array}{l}\text { 'Diese Informationen stehen in jeweils aktualisierter Form auf der Home- } \\
\text { page der ÖGARI (www.oegari.at) und als App für Smartphones unter app. } \\
\text { oegari.at zur Verfügung } \\
\text { bln prophylaktischer Dosierung } \\
\text { cLaut Hersteller ist das Setzen eines Epiduralkatheters unter Dabigatran } \\
\text { kontraindiziert }\end{array}$} \\
\hline
\end{tabular}

Mittlerweile existieren auf österreichischer [58], aber auch auf europäischer Ebene, [59] Empfehlungen zum Umgang mit Regionalanästhesie bei Antikoagulation. Da es dazu so gut wie keine Evidenz gibt, beruhen diese Empfehlungen im Wesentlichen auf der Pharmakologie der jeweiligen gerinnungshemmenden Substanzen und daraus abgeleiteten Erwägungen.

Grundsätzlich sind als blutungsrelevante Interventionen nicht nur die Punktion, sondern auch die Anlage und die Entfernung eines Epiduralkatheters zu betrachten.

Präoperativ sind Risikostratifizierung, Aufklärung und Einwilligung des Patienten sowie eine entsprechende Medikamentenverordnung bzw. auch das Absetzen der Antikoagulation (s. Tab. 6) wichtig.

Von entscheidender Bedeutung ist der Zeitpunkt der Regionalanästhesie - sie sollte erst erfolgen, wenn die gerinnungshemmende Wirkung des jeweiligen Antikoagulans mit größter Wahrscheinlichkeit abgeklungen ist. Tabelle 6 gibt einen Überblick der einzuhaltenden Wartezeiten von der letzten Dosis eines Antikoagulans bis zur neuroaxialen Anästhesie und der Zeiten bis zur nächsten Folgedosis.

Allerdings sind Faktoren wie das individuelle Erreichen des Talspiegels und Veränderungen der Pharmakokinetik durch Medikamenteninteraktionen oder Störungen der Leber- oder Nierenfunktion in den in Tab. 6 angegebenen Daten nicht berücksichtigt.

Aus der Sicht der neuroaxialen Anästhesie ist die Tatsache, dass eine VTE-Prophylaxe mit DOAK oder Fondaparinux grundsätzlich postoperativ begonnen wird, als Vorteil zu sehen, der allerdings bei Reoperationen und bei der Entfernung eines Epiduralkatheters wegfällt.

Intraoperativ sind die Technik der Regionalanästhesie und die Gerinnungsoptimierung die wichtigsten Faktoren, postoperativ geht es um Vigilanz hinsichtlich spinaler Blutungen und rascher Intervention bei dementsprechendem Verdacht.

Das Management DOAK-induzierter Blutungen wurde an anderer Stelle ausführlich beschrieben [60]. Es 
sei hier jedoch erwähnt, dass die Österreichische Gesellschaft für Anästhesiologie, Reanimation und Intensivmedizin (ÖGARI) ein Register zu Blutungen unter DOAK eingerichtet hat. Meldungen werden unter der Adresse http://178.77.73.162/share/DOAK/form/forms_public/ DOAK_line.html erbeten.

\section{Empfehlungen zu Antikoagulation und neuroaxiale Blockaden}

Traumatische Punktionen sollten möglichst vermieden werden.

Bei Blutaspiration und geplanter intraoperativer Antikoagulation sollte eine Verschiebung des Eingriffs erwogen werden [61].

Eine individuelle Nutzen-Risiko-Abwägung ist unbedingt erforderlich. Wenn eine entsprechende Wartezeit nach Absetzen des Antikoagulans nicht möglich ist, sollten Alternativen zur neuroaxialen Anästhesie überlegt werden [62].

Eine entsprechende interdisziplinäre postoperative Überwachung ist fixer Bestandteil des Risikomanagements.

Symptome, die den Verdacht auf ein spinales Hämatom nahelegen, sind persistierende sensomotorische Defizite, radikulärer Rückenschmerz und Blasenfunktionsstörung. In einem solchen Fall ist eine sofortige Bildgebung (MRT, CT) und ggf. sofortige Therapie (Laminektomie) zu veranlassen [57].

\section{Bridging}

Für das oft als „Bridging“ bezeichnete perioperative Management antikoagulierter Patienten ist es notwendig, das Thromboserisiko (und zwar sowohl das vorbestehende als Grund der Antikoagulation als das zusätzlich durch den geplanten Eingriff zu erwartende) dem durch Antikoagulation erhöhten Blutungsrisiko während des Eingriffs und danach gegenüberzustellen. Steht das erhöhte Blutungsrisiko im Vordergrund, muss die antithrombotische Therapie abgesetzt werden. Steht das Thromboserisiko im Vordergrund, ist eine Überbrückungstherapie notwendig.

Tab. 7 Einteilung des VTE-Risikos bei Dauerantikoagulation. (Quelle: [63])

\begin{tabular}{|c|c|c|c|c|}
\hline $\begin{array}{l}\text { VTE- } \\
\text { Risiko }\end{array}$ & $\begin{array}{l}\text { VHF (Punkte im } \\
\left.\text { CHADS }_{2} \text {-Score }{ }^{a}\right)\end{array}$ & $\begin{array}{l}\text { Mechanische } \\
\text { Herzklappen }\end{array}$ & VTE & Bridging \\
\hline \multirow[t]{3}{*}{ Hoch } & \multirow[t]{3}{*}{$5-6$} & Künstliche MK & \multirow[t]{3}{*}{$<3$ Mo } & \multirow[t]{4}{*}{$J A$} \\
\hline & & $\begin{array}{l}\text { Ältere künstliche } \\
\text { AK }\end{array}$ & & \\
\hline & & Insult/TIA < 3 Mo & & \\
\hline Mittel & $3-4$ & DFAK mit RF & $>3$ bis $12 \mathrm{Mo}$ & \\
\hline Niedrig & $0-2$ & $\begin{array}{l}\text { DFAK ohne RF, alle } \\
\text { Bioklappen }\end{array}$ & $>12 \mathrm{Mo}$ & NEIN \\
\hline
\end{tabular}

aDer $\mathrm{CHADS}_{2}$-Score beruht auf den fünf wichtigsten Insult-Risikofaktoren: Herzinsuffizienz, Hypertonie, Alter $\geq 75 a$, Diabetes und früherer Insult $[64,65]$

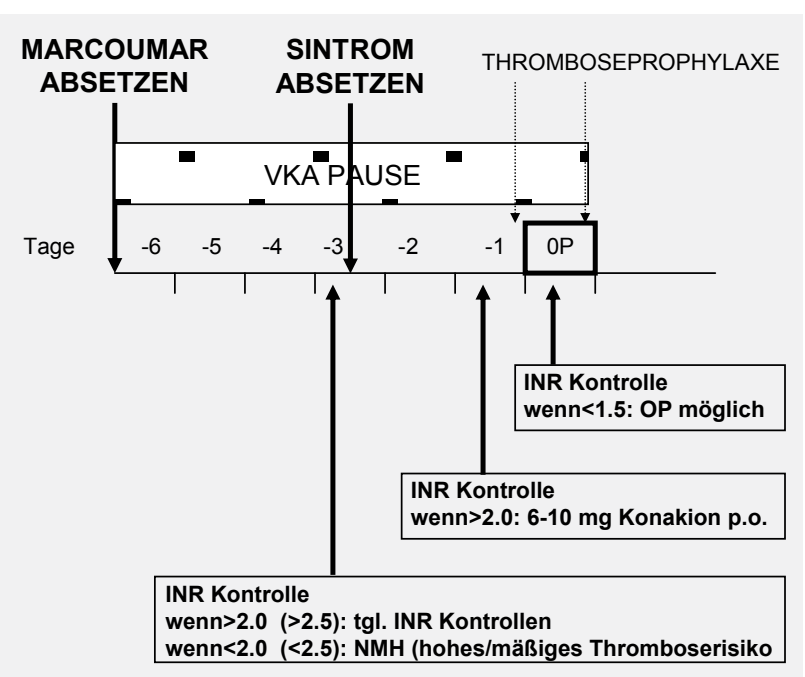

Abb. 1 Präoperatives Vorgehen bei Absetzen von VKA. (Quelle: Modifiziert nach Watzke $\mathrm{H}$ von Eichinger $\mathrm{S}$, beide UK $\mathrm{f}$. Innere Medizin I, MUW)

Anhand der wichtigsten Indikationen für eine Dauerantikoagulation (Vorhofflimmern, Herzklappenersatz, venöse Thromboembolie) lässt sich das VTE-Risiko in drei Kategorien - hoch, mittel, niedrig - einteilen (s. Tab. 7) [63].

$\mathrm{Zu}$ den folgenden Empfehlungen ist zu sagen, dass sie ausnahmslos nicht auf Ergebnissen kontrollierter Studien beruhen, sondern Beobachtungen in Patientenserien bzw. Expertenmeinungen widerspiegeln. Patienten mit niedrigem VTE-Risiko benötigen keine Überbrückungstherapie. Für Patienten mit mittlerem oder hohem Risiko wird hingegen ein Bridging empfohlen.

Zumeist wird für die Überbrückungstherapie ein NMH verwendet, für Patienten mit hohem Risiko wird die therapeutische Dosis empfohlen. Bei Patienten mit niedrigem Risiko liegt es im Ermessen des Behandlers, $\mathrm{NMH}$ in therapeutischer oder prophylaktischer Dosis einzusetzen. Im Zweifelsfall sollte die therapeutische Dosis verwendet werden.

Eingriffe mit niedrigem Blutungsrisiko (z. B. unkomplizierte Zahnextraktionen oder kleine dermatologische Eingriffe) können unter laufender Antikoagulation durchgeführt werden - dies gilt auch für Patienten, die mit DOAK behandelt werden.

Wenn ein VKA abgesetzt werden muss, sollte dies nicht zu früh geschehen (s. Abb. 1). Andererseits darf, um das Blutungsrisiko nicht zu stark zu erhöhen, das NMH nicht zu früh nach Absetzen des VKA begonnen werden. Die letzte therapeutische NMH-Dosis sollte $24 \mathrm{~h}$ vor der Operation verabreicht werden. Ein Schema für das präoperative Vorgehen zeigt Abb. 1.

Postoperativ sollte ein NMH in therapeutischer Dosis frühestens $48 \mathrm{~h}$ nach dem Eingriff verabreicht werden. Ein Schema für das postoperative Vorgehen gibt Abb. 2.

Im Gegensatz zu den Bridging-Empfehlungen selbst, ist die Tatsache, dass übermäßiges bzw. nicht fachgerecht durchgeführtes Bridging das Blutungsrisiko sowohl 
Tab. 8 Zeitpunkt für das Absetzen von Dabigatran vor einem elektivem Eingriff. (Quelle: [4])

\begin{tabular}{l|l|l|l|}
$\begin{array}{l}\text { Nierenfunktion } \\
(\mathrm{KrCl} \mathrm{ml} / \mathrm{min})\end{array}$ & HWZ (h) & $\begin{array}{l}\text { Hohes Blutungsrisiko } \\
\text { oder größerer Eingriff }\end{array}$ & \multicolumn{2}{l}{ Standardrisiko } \\
\hline$\geq 80$ & 13 & 2d vorher & 24 h vorher \\
\hline$\geq 50-<80$ & 15 & 2-3d vorher & 1-2d vorher \\
\hline$\geq 30-<50$ & 18 & 4d vorher & 2-3d vorher $(>48 \mathrm{~h})$ \\
\hline
\end{tabular}

intra- als auch postoperativ erhöht, sehr wohl durch Daten belegbar [66].

Der Wiederbeginn einer VKA-Behandlung nach einem Eingriff darf keinesfalls nach dem Standardschema einer Neueinstellung erfolgen, weil dies bei manchen Patienten zu Überdosierungen führen könnte. Vielmehr muss unter Bedachtnahme auf die bekannte Vordosierung und auf eine höhere postoperative Empfindlichkeit gegenüber VKA - mit niedrigen Dosen begonnen und langsam gesteigert werden. Dies gilt insbesondere natürlich dann, wenn der Patient zuvor schon eine niedrige VKAErhaltungsdosis gebraucht hat.

Wenn ein Patient therapeutische Dosen von DOAK (z. B. bei Vorhofflimmern) erhält, so können Eingriffe mit niedrigem Blutungsrisiko unter laufender DOAK-Therapie durchgeführt werden. Allerdings ist auch in diesem Fall zu empfehlen, das DOAK am Tag des Eingriffs erst einige Stunden nach diesem zu verabreichen.

Ist das Blutungsrisiko höher, muss das DOAK präoperativ abgesetzt werden.

Aufgrund der kurzen Halbwertszeit von DOAK wird ein Bridging mit NMH vor dem Eingriff nicht als erforderlich betrachtet [67].

Der Zeitpunkt des Absetzens hängt für Dabigatran jedoch wegen der hohen renalen Ausscheidungsrate von der Nierenfunktion ab (Details siehe Tab. 8) [4].

Wenn eine Indikation für die postoperative Prophylaxe mit Dabigatran gegeben ist, so kann diese nach üblichem Schema beginnen. Ist dies nicht der Fall, so muss eine andere, übliche VTE-Prophylaxe (zumeist also mit $\mathrm{NMH}$ ) erfolgen, bis Dabigatran wieder in therapeutischer Dosis verabreicht werden kann - dies entspricht dem Vorgehen bei Wiederbeginn einer Antikoagulation mit VKA oder NMH in therapeutischer Dosis (siehe Abb. 2). Für das perioperative Vorgehen bei Patienten unter Dabigatran gibt es auch rezente österreichische Konsensusempfehlungen [68].

Das Absetzen von Rivaroxaban bzw. Apixaban sollte $24 \mathrm{~h}$, gegebenenfalls $48 \mathrm{~h}$ (hohes Blutungsrisiko bzw. Niereninsuffizienz) vor dem Eingriff erfolgen [2, 3]. Auch hier ist kein präoperatives Bridging erforderlich. Für das Vorgehen in der postoperativen Phase gilt analog das für Dabigatran Gesagte.

Für das Vorgehen bei intraartikulären Injektionen gibt es keine kontrollierten Studien. Die DOAK-Einnahme am Tag der intraartikulären Injektion ist auf den Abend zu verschieben - in Analogie zur Entfernung intraspinaler Katheter. Am Morgen des Tages nach dem Eingriff kann das DOAK normal weiter verabreicht werden.

An dieser Stelle sei nur kurz erwähnt, dass es - bei eher schlechter Datenlage - auch bereits Bridging-Empfehlungen für Patienten unter Thrombozytenfunktions-hemmender Therapie gibt [69]. Zu betonen ist, dass weder NMH noch DOAK für ein solches Bridging geeignet sind.

\section{Empfehlungen zu Bridging}

Die Entscheidung für oder gegen eine Bridging-Therapie hängt von der Abwägung des Thromboserisikos gegen das Blutungsrisiko, von der Indikation für die Antikoagulation und vom dafür verwendeten Medikament ab.

Im Allgemeinen werden mit VKA dauerantikoagulierte Patienten vor operativen Eingriffen dann mit Bridging (zumeist NMH) behandelt, wenn sie ein mittleres oder hohes Thromboembolierisiko aufweisen.
Abb. 2 Postoperatives Vorgehen nach VKA-Unterbrechung. (Quelle: Modifiziert nach Watzke $\mathrm{H}$ von Eichinger $\mathrm{S}$, beide UK f. Innere Medizin I, MUW)

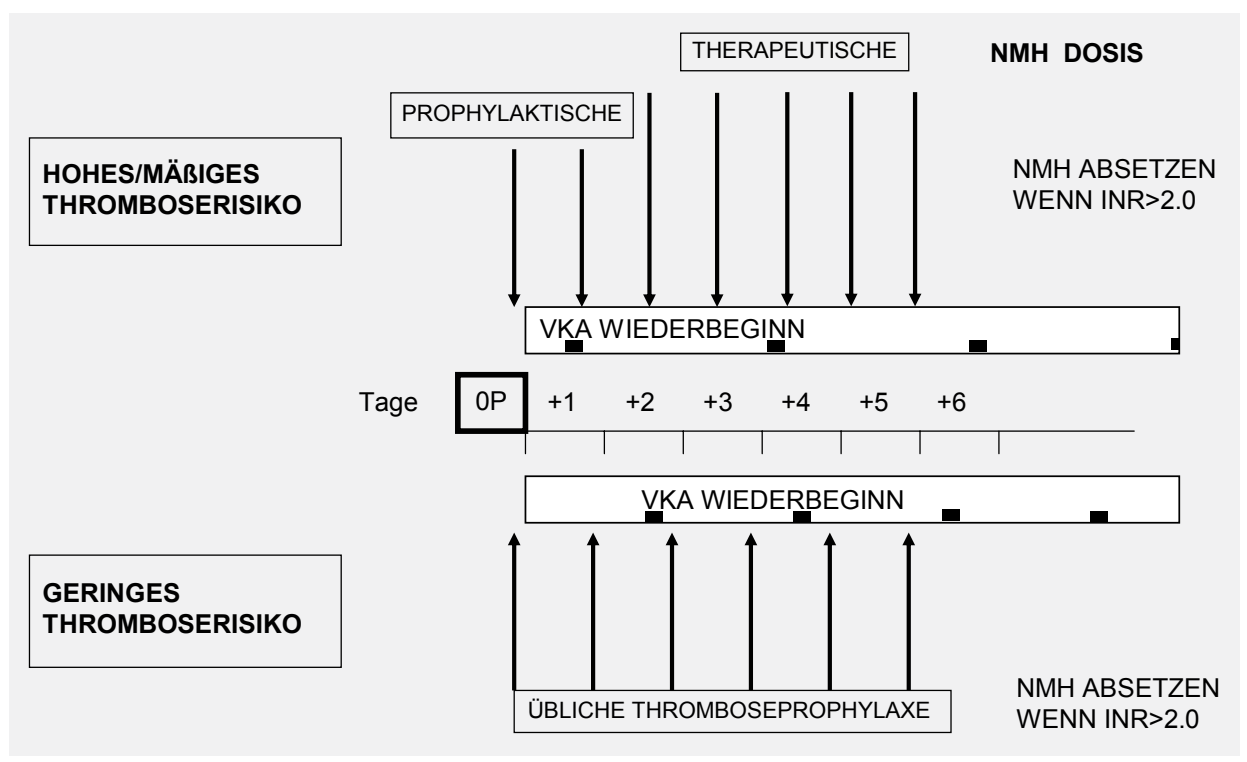


Bei therapeutischem Einsatz von DOAK, Fondaparinux oder $\mathrm{NMH}$ ist aufgrund der kurzen Halbwertszeiten vor dem Eingriff meist kein Bridging erforderlich.

Bei Dabigatran ist wegen der Veränderung der HWZ die Nierenfunktion zu beachten.

Am Tag der Operation sollten - außer in ganz ausgewählten Fällen eines extrem hohen Risikos - ausschließlich Prophylaxedosen von Antikoagulanzien verabreicht werden.

\section{Interessenskonflikte}

Die technische Durchführung des Konsensusmeetings und des nachfolgenden Abstimmungsprozesses sowie die Publikation dieses Konsensusstatements wurden durch Unresctricted Grants der Firmen Bayer, Boehringer Ingelheim und Pfizer an die Österreichische Gesellschaft für Orthopädie und Orthopädische Chirurgie (ÖGO) ermöglicht.

Open Access Dieser Artikel unterliegt den Bedingungen der Creative Commons Attribution Noncommercial License. Dadurch sind die nichtkommerzielle Nutzung, Verteilung und Reproduktion erlaubt, sofern der/die Originalautor/en und die Quelle angegeben sind.

\section{Literatur}

1. Falck-Ytter Y, et al. Prevention of VTE in orthopedic surgery patients: antithrombotic therapy and prevention of thrombosis, 9th ed: American College of Chest Physicians Evidence-Based Clinical Practice Guidelines. Chest. 2012;141(2 Suppl.):e278S-325S.

2. Austria Codex online. Fachinformation Eliquis ${ }^{\circledR}$ Filmtabletten. 2012. www.pharmazie.com. Zugegriffen: 17. Jan. 2013.

3. Austria Codex online. Fachinformation Xarelto ${ }^{\circledR}$ Filmtabletten. 2012. www.pharmazie.com. Zugegriffen: 17. Jan. 2013.

4. Austria Codex online. Fachinformation Pradaxa ${ }^{\circledR}$ Hartkapseln. 2012. www.pharmazie.com. Zugegriffen: 17. Jan. 2013.

5. Austria Codex online. Fachinformation Arixtra ${ }^{\circledR}$ Injektionslösung. 2012. www.pharmazie.com. Zugegriffen: 17. Jan. 2013.

6. Clemens A, et al. Switching from enoxaparin to dabigatran etexilate: pharmacokinetics, pharmacodynamics, and safety profile. Eur J Clin Pharmacol. 2012;68(5):607-16.

7. Eerenberg ES, et al. Reversal of rivaroxaban and dabigatran by prothrombin complex concentrate: a randomized, placebo-controlled, crossover study in healthy subjects. Circulation. 2011;124(14):1573-9.

8. Kaatz S, et al. Guidance on the emergent reversal of oral thrombin and factor Xa inhibitors. Am J Hematol. 2012;87 (Suppl. 1):141-5.

9. Fries D, et al. Management of dabigatran-induced bleeding: expert statement. Wien Klin Wochenschr. 2013;125(21-22):721-9.

10. Pabinger I, et al. [Austrian Guidelines for Prophylaxis of Venous Thromboembolism]. Wien Klin Wochenschr. 2007;119(23-24):739-46.

11. White $\mathrm{RH}$, et al. Incidence and time course of thromboembolic outcomes following total hip or knee arthroplasty. Arch Intern Med. 1998;158(14):1525-31.
12. Bjornara BT, et al. Frequency and timing of clinical venous thromboembolism after major joint surgery. J Bone Joint Surg Br. 2006;88(3):386-91.

13. Eikelboom JW, et al. Extended-duration prophylaxis against venous thromboembolism after total hip or knee replacement: a meta-analysis of the randomised trials. Lancet. 2001;358(9275):9-15.

14. Hull RD, et al. Extended out-of-hospital low-molecularweight heparin prophylaxis against deep venous thrombosis in patients after elective hip arthroplasty: a systematic review. Ann Intern Med. 2001;135(10):858-69.

15. O'Donnell M, et al. Reduction of out-of-hospital symptomatic venous thromboembolism by extended thromboprophylaxis with low-molecular-weight heparin following elective hip arthroplasty: a systematic review. Arch Intern Med. 2003;163(11):1362-6.

16. Hui AC, et al. Graded compression stockings for prevention of deep-vein thrombosis after hip and knee replacement. J Bone Joint Surg Br. 1996;78(4):550-4.

17. Muir KW, et al. Randomized trial of graded compression stockings for prevention of deep-vein thrombosis after acute stroke. QJM. 2000;93(6):359-64.

18. Dennis M, et al. Effectiveness of thigh-length graduated compression stockings to reduce the risk of deep vein thrombosis after stroke (CLOTS trial 1): a multicentre, randomised controlled trial. Lancet. 2009;373(9679):1958-65.

19. Nieto JA, et al. Dabigatran, rivaroxaban and apixaban versus enoxaparin for thomboprophylaxis after total knee or hip arthroplasty: pool-analysis of phase III randomized clinical trials. Thromb Res. 2012.

20. Lassen MR, et al. Apixaban or enoxaparin for thromboprophylaxis after knee replacement. N Engl J Med. 2009;361(6):594-604.

21. Lassen MR, et al. Apixaban versus enoxaparin for thromboprophylaxis after knee replacement (ADVANCE-2): a randomised double-blind trial. Lancet. 2010;375(9717):807-15.

22. Eriksson BI, et al. Oral dabigatran etexilate vs. subcutaneous enoxaparin for the prevention of venous thromboembolism after total knee replacement: the RE-MODEL randomized trial. J Thromb Haemost. 2007;5(11):2178-85.

23. Ginsberg JS, et al. Oral thrombin inhibitor dabigatran etexilate vs North American enoxaparin regimen for prevention of venous thromboembolism after knee arthroplasty surgery. J Arthroplasty. 2009;24(1):1-9.

24. Huisman MV, et al. Enoxaparin versus dabigatran or rivaroxaban for thromboprophylaxis after hip or knee arthroplasty: results of separate pooled analyses of phase III multicenter randomized trials. Circ Cardiovasc Qual Outcomes. 2010;3(6):652-60.

25. Lassen MR, et al. Rivaroxaban versus enoxaparin for thromboprophylaxis after total knee arthroplasty. N Engl J Med. 2008;358(26):2776-86.

26. Turpie AG, et al. Rivaroxaban versus enoxaparin for thromboprophylaxis after total knee arthroplasty (RECORD4): a randomised trial. Lancet. 2009;373(9676):1673-80.

27. Lassen MR, et al. Apixaban versus enoxaparin for thromboprophylaxis after hip replacement. $\mathrm{N}$ Engl J Med. 2010;363(26):2487-98.

28. Eriksson BI, et al. Dabigatran etexilate versus enoxaparin for prevention of venous thromboembolism after total hip replacement: a randomised, double-blind, non-inferiority trial. Lancet. 2007;370(9591):949-56.

29. Eriksson BI, et al. Oral dabigatran versus enoxaparin for thromboprophylaxis after primary total hip arthroplasty (RE-NOVATE II*). A randomised, double-blind, non-inferiority trial. Thromb Haemost. 2011;105(4):721-9. 
30. Eriksson BI, et al. Novel oral factor Xa and thrombin inhibitors in the management of thromboembolism. Annu Rev Med. 2011;62:41-57.

31. Eriksson BI, et al. Rivaroxaban versus enoxaparin for thromboprophylaxis after hip arthroplasty. N Engl J Med. 2008;358(26):2765-75.

32. Kakkar AK, et al. Extended duration rivaroxaban versus short-term enoxaparin for the prevention of venous thromboembolism after total hip arthroplasty: a double-blind, randomised controlled trial. Lancet. 2008;372(9632):31-9.

33. Eriksson BI, et al. Duration of prophylaxis against venous thromboembolism with fondaparinux after hip fracture surgery: a multicenter, randomized, placebo-controlled, double-blind study. Arch Intern Med. 2003;163(11):1337-42.

34. Eriksson BI, et al. Fondaparinux compared with enoxaparin for the prevention of venous thromboembolism after hipfracture surgery. N Engl J Med. 2001;345(18):1298-1304.

35. Turner JA, et al. Patient outcomes after lumbar spinal fusions. JAMA. 1992;268(7):907-11.

36. Geerts WH, et al. Prevention of venous thromboembolism. Chest. 2001;119(1 Suppl.):132S-175S.

37. Agnelli G. Prevention of venous thromboembolism in surgical patients. Circulation. 2004;110(24 Suppl. 1):IV4-12.

38. Oda T, et al. Deep venous thrombosis after posterior spinal surgery. Spine (Phila Pa 1976). 2000;25(22):2962-7.

39. Collen JF, et al. Prevention of venous thromboembolism in neurosurgery: a metaanalysis. Chest. 2008;134(2):237-49.

40. Gould MK, et al. Prevention of VTE in nonorthopedic surgical patients: antithrombotic therapy and prevention of thrombosis, 9th ed: American College of Chest Physicians Evidence-Based Clinical Practice Guidelines. Chest. 2012;141(2 Suppl.):e227S-77S.

41. Dennis JW, et al. Efficacy of deep venous thrombosis prophylaxis in trauma patients and identification of high-risk groups. J Trauma. 1993;35(1):132-8; discussion 138-9.

42. Arbeitsgruppe für Thromboseprophylaxe der AUVA. Klinischer Pfad "Thromboseprophylaxe" innerhalb der AUVA (QA 14). 2004-2005. http://www.auva.at/mediaDB/965213_Klinischer_Pfad_Thromboseprophylaxe.pdf. Zugegriffen: 8. April 2013.

43. Ramos J, et al. Interventions for preventing venous thromboembolism in adults undergoing knee arthroscopy. Cochrane Database Syst Rev. 2008(4):CD005259.

44. Camporese G, et al. Low-molecular-weight heparin versus compression stockings for thromboprophylaxis after knee arthroscopy: a randomized trial. Ann Intern Med. 2008;149(2):73-82.

45. Marlovits S, et al. Extended-duration thromboprophylaxis with enoxaparin after arthroscopic surgery of the anterior cruciate ligament: a prospective, randomized, placebocontrolled study. Arthroscopy. 2007;23(7):696-702.

46. Testroote M, et al. Low molecular weight heparin for prevention of venous thromboembolism in patients with lower-leg immobilization. Cochrane Database Syst Rev. 2008(4):CD006681.

47. Tryba M. [Epidural regional anesthesia and low molecular heparin: pro]. Anasthesiol Intensivmed Notfallmed Schmerzther. 1993;28(3):179-81.

48. Schroeder DR. Statistics: detecting a rare adverse drug reaction using spontaneous reports. Reg Anesth Pain Med. 1998;23(6 Suppl. 2):183-9.

49. Moen V, et al. Severe neurological complications after central neuraxial blockades in Sweden 1990-1999. Anesthesiology. 2004;101(4):950-9.

50. Stafford-Smith M. Impaired haemostasis and regional anaesthesia. Can J Anaesth. 1996;43(5 Pt 2):R129-41.
51. Lee LA, et al. Injuries associated with regional anesthesia in the 1980s and 1990s: a closed claims analysis. Anesthesiology. 2004;101(1):143-52.

52. Cameron CM, et al. A review of neuraxial epidural morbidity: experience of more than 8,000 cases at a single teaching hospital. Anesthesiology. 2007;106(5):997-1002.

53. Christie IW, et al. Major complications of epidural analgesia after surgery: results of a six-year survey. Anaesthesia. 2007;62(4):335-41.

54. Popping DM, et al. Effectiveness and safety of postoperative pain management: a survey of 18925 consecutive patients between 1998 and 2006 (2nd revision): a database analysis of prospectively raised data. $\mathrm{Br} \mathrm{J}$ Anaesth. 2008;101(6):832-40.

55. Cook TM, et al. Major complications of central neuraxial block: report on the Third National Audit Project of the Royal College of Anaesthetists. Br J Anaesth. 2009;102(2):179-90.

56. Volk T, et al. Incidence of spinal haematoma after epidural puncture: analysis from the German network for safety in regional anaesthesia. Eur J Anaesthesiol. 2012;29(4):170-6.

57. Vandermeulen EP, et al. Anticoagulants and spinal-epidural anesthesia. Anesth Analg. 1994;79(6):1165-77.

58. Arbeitsgruppe perioperative Gerinnung der ÖGARI. Regionalanästhesie unter gerinnungshemmender Medikation. 2012. http://www.oegari.at/web_files/dateiarchiv/116/Empfehlungen\%20Regionalan\%C3\%A4sthesie\%20unter\%20Gerinnungshemmung\%202012.pdf. Zugegriffen: 24. Juni 2012.

59. Gogarten W, et al. Regional anaesthesia and antithrombotic agents: recommendations of the European Society of Anaesthesiology. Eur J Anaesthesiol. 2010;27(12):999-1015.

60. Kozek-Langenecker S. Management der DOAK-induzierten Blutung. In: Intensivmedizin - Kompendium und Repetitorium zur interdisziplinären Weiter- und Fortbildung, Eckhart J, Forst H, Briegel J, Herausgeber. ecomed MEDIZIN. 2012. ISBN 978-3-609-20210-5.

61. Chaney MA. Intrathecal and epidural anesthesia and analgesia for cardiac surgery. Anesth Analg. 1997;84(6):1211-21.

62. Chaney MA. Cardiac surgery and intrathecal/epidural techniques: at the crossroads? Can J Anaesth. 2005;52(8):783-8.

63. Douketis JD. Perioperative management of patients who are receiving warfarin therapy: an evidence-based and practical approach. Blood. 2011;117(19):5044-9.

64. Gage BF et al. Selecting patients with atrial fibrillation for anticoagulation: stroke risk stratification in patients taking aspirin. Circulation. 2004;110(16):2287-92.

65. Kearon C, et al. Management of anticoagulation before and after elective surgery. N Engl J Med. 1997;336(21):1506-11.

66. Kovacs MJ, et al. Single-arm study of bridging therapy with low-molecular-weight heparin for patients at risk of arterial embolism who require temporary interruption of warfarin. Circulation. 2004;110(12):1658-63.

67. Healey JS, et al. Periprocedural bleeding and thromboembolic events with dabigatran compared with warfarin: results from the Randomized Evaluation of Long-Term Anticoagulation Therapy (RE-LY) randomized trial. Circulation. 2012;126(3):343-8.

68. Weltermann A, et al. Dabigatran in patients with atrial fibrillation: perioperative and periinterventional management. Wien Klin Wochenschr. 2012;124(9-10):340-7.

69. Korte $\mathrm{W}$, et al. Peri-operative management of antiplatelet therapy in patients with coronary artery disease: joint position paper by members of the working group on Perioperative Haemostasis of the Society on Thrombosis and Haemostasis Research (GTH), the working group on Perioperative Coagulation of the Austrian Society for Anesthesiology, Resuscitation and Intensive Care (OGARI) and the Working Group Thrombosis of the European Society for Cardiology (ESC). Thromb Haemost. 2011;105(5):743-9. 